\title{
The emergent diseases in grapevine nursery: Critical point analysis for developing a protected and sustainable propagation process
}

\author{
E. Battiston ${ }^{1}$, S. Di Marco ${ }^{2}$, E. Angelini ${ }^{3}$, and L. Mugnai ${ }^{1}$ \\ ${ }^{1}$ University of Florence, DAGRI, 50144 Firenze, Italy \\ ${ }^{2}$ National Research Council, IBIMET, 40129 Bologna, Italy \\ ${ }^{3}$ CREA, Research Center for Viticulture and Enology, 31010 Conegliano, Italy
}

\begin{abstract}
The diffusion of emergent pathogens in grapevine nursery is increasingly compromising the sanitary quality of the propagation material. The complexity of the problem lies on the plurality of plant diseases involved and on the high environmental and economic impact of the conventional methods applied to comply the phytosanitary standards. By a critical point analysis, the study suggests the development of nursery strategies focused on the sanitary quality of the production and on its long-term environmental sustainability.
\end{abstract}

\section{Introduction}

Nowadays viticulture is called upon to make decisions that respond not only to quality and productivity, but also to sustainability and sanitary protection, developing modern strategies of cultivation and propagation with the least environmental impact and the best efficiency. In the meantime, the growing spread of complex diseases all along the grapevine production chain suggests to take into consideration even the vine propagation stage, through which multiple pathogens can potentially spread significantly.

In grapevine nurseries, emerging plant pathogens are increasingly compromising the sanitary quality of the propagation material and the complexity of the problem lies on the plurality of the pathogens involved: grapevine trunk diseases, bacterioses, and viruses. In addition, the methodologies and tools conventionally used to guarantee the minimal sanitary standards required by the current legislation show a certain fragility and are in any case particularly impacting the nursery chain, by the environmental and economic standpoints.

\section{Materials and methods}

The study was aimed at a critical point analysis, focusing on: (i) the risk of contamination and spread of emerging grapevine pathogens; (ii) the unreliability of traditional methods in controlling such pathogens; (iii) the compatibility of efficient disease management strategies with the standards for organic agriculture.

The following critical points are analyzed: origin and selection of scions and rootstocks, storage and sanitization of propagation material, grafting methods, disease management in the propagation stage and in the nursery field. The analysis was performed considering the incidence of the following grapevine diseases: grapevine trunk diseases, grapevine crown gall and rugose wood complex.

\section{Results}

The study highlights the possibility to improve the management of grapevine trunk diseases by acting on the training system for rootstocks, the storage conditions of rootstocks and scions and their treatment with sanitizers allowed in organic agriculture, on the type of grafting, on the substrates and conditions for callusing. More specifically, organic treatments are also potentially efficient on the management of grapevine crown gall. A reliable and sustainable control of viruses would be achieved by developing an innovative protocol for the material selection in mother blocks, investigating also the scion/rootstock graft incompatibility related to the presence of the rugose wood complex viruses.

\section{Conclusions}

The study defines a framework that offers the opportunity to develop nursery strategies, focusing mainly on the sanitary quality of the production and on its long-term environmental sustainability.

\section{References}

The study is part of the OIV Research Grant Program 2018 and the VITISBIO ${ }^{\circledast}$ project (funds EAFRD 2014-2020, region Friuli Venezia Giulia, Italy). 